\title{
The Virtual Mitten: A Novel Interaction Paradigm for Visuo-Haptic Manipulation of Objects Using Grip Force
}

\author{
Merwan Achibet* \\ Inria Rennes, France
}

\author{
Maud Marchal* \\ INSA/Inria Rennes, France
}

\author{
Ferran Argelaguet* \\ Inria Rennes, France
}

Anatole Lécuyer*
Inria Rennes, France

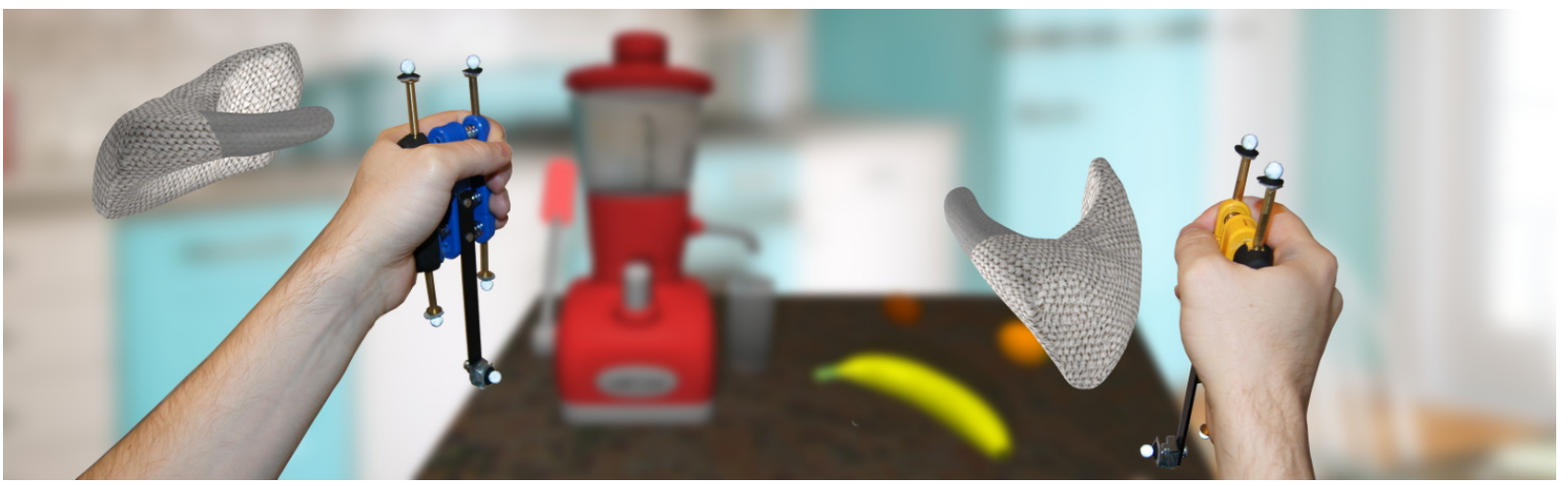

Figure 1: Visuo-haptic manipulation as enabled by our novel approach called the "Virtual Mitten". Each hand holds an elastic device to control a corresponding virtual mitten (in gray) and to grasp virtual objects in a bimanual scenario. The grip force applied by the user is measured to generate pseudo-haptic feedback.

\begin{abstract}
In this paper, we propose a novel visuo-haptic interaction paradigm called the "Virtual Mitten" for simulating the 3D manipulation of objects. Our approach introduces an elastic handheld device that provides a passive haptic feedback through the fingers and a mitten interaction metaphor that enables to grasp and manipulate objects. The grasping performed by the mitten is directly correlated with the grip force applied on the elastic device and a supplementary pseudo-haptic feedback modulates the visual feedback of the interaction in order to simulate different haptic perceptions. The Virtual Mitten allows natural interaction and grants users with an extended freedom of movement compared with rigid devices with limited workspaces. Our approach has been evaluated within two experiments focusing both on subjective appreciation and perception. Our results show that participants were able to well perceive different levels of effort during basic manipulation tasks thanks to our pseudo-haptic approach. They could also rapidly appreciate how to achieve different actions with the Virtual Mitten such as opening a drawer or pulling a lever. Taken together, our results suggest that our novel interaction paradigm could be used in a wide range of applications involving one or two-hand haptic manipulation such as virtual prototyping, virtual training or video games.
\end{abstract}

Index Terms: H.5.2 [Information Interfaces and Presentation]: User Interfaces-Direct manipulation, Haptic I/O;

\section{INTRODUCTION}

Object manipulation is a fundamental task in virtual reality applications [7]. Several methods have been proposed to grab and manipulate virtual objects by moving hands in $3 \mathrm{D}$ space. These tech-

\footnotetext{
*\{merwan.achibet, maud.marchal, fernando.argelaguet_sanz, anatole.lecuyer\}@inria.fr
}

niques may rely on optical tracking [26] but the anatomical complexity of the human hand makes the accurate tracking of manual gestures a difficult task and haptic feedback, which is an important cue in object manipulation, is missing. The simulation of manipulation tasks can then be enhanced with haptic interfaces, which aim at generating sensations related to the sense of touch and effort. Force-feedback arms enable to manipulate virtual objects and generate interaction forces towards the user but generally require specific interaction metaphors that do not always reproduce the natural dynamics of grasping. Active hand-mounted devices enable to accurately track the hand and to feel virtual objects with the fingers [6] but they remain rather complex and costly. Although alternative means of haptic stimulation have been proposed (e.g. vibrotactile feedback or passive haptic feedback), they seem currently limited for providing a convincing haptic perception in the context of manipulation tasks, especially for grasping.

In this paper, we aim at proposing a novel interaction paradigm to naturally grasp and manipulate virtual objects with haptic sensations: the Virtual Mitten. This paradigm is based on a handheld elastic device that allows us to control a virtual mitten in the simulation. Upon clenching actions from the user, the virtual mitten can be operated in 3D space to grasp or release virtual objects. A pseudo-haptic effect that takes as input the user's grip force is added to simulate modulated levels of effort when performing manipulation tasks. Our contributions can be summarized as follows:

- the introduction of a low-cost handheld input device that generates elastic feedback related to grip force and preserves freedom of movement within the virtual environment due to its low weight and small size,

- an interaction metaphor that takes the form of a mitten bound to a control scheme that allows users to manipulate virtual objects through natural grasping motions and grip forces,

- a novel pseudo-haptic approach based on grip force to vary the perceived effort when performing object manipulation tasks. 
In the remainder of this paper, we first present in Section 2 an overview of related work in $3 \mathrm{D}$ visuo-haptic manipulation of objects. Then, the concept of the Virtual Mitten is detailed in Section 3. In Section 4, a user study conducted to evaluate its appeal as an interaction paradigm as well as its novel pseudo-haptic approach is described. The paper ends with a general discussion in Section 5 and a conclusion.

\section{Related work}

In this section, we present an overview of the state of the art related to $3 \mathrm{D}$ visuo-haptic manipulation of virtual objects. We cover taxonomies of manipulation patterns, hand-based manipulation techniques, active haptic devices and alternative methods to provide haptic feedback.

\subsection{Taxonomies of hand-based interaction}

The hand is a high-dimensional organ which mechanical properties allow many configurations and types of interaction. However, only a small subset of manipulation patterns are consistently used. Classifications have thus been developed in order to formalize the way that we, as humans, interact with our environment. Early work focused on grasping patterns and precise finger configurations $[28,22]$. However, recent taxonomies have taken a more functional approach. For example, Bullock et al. [8] adopted a hand-centric view to classify hand-based interaction and proposed to differentiate them according to criteria such as prehension/non-prehension, global motion or motion of the object with respect to the hand. Bloomfield et al. [3] established a similar classification but from a force-centric perspective and specifically oriented towards haptic tasks for virtual reality. Dexterous actions are here classified according to the mechanical forces that they involve:

- Force I: the applied force is aligned with the motion (e.g. pushing a box).

- Force II: the applied force is not aligned with the motion (e.g. pulling a pin by gripping its head).

- Torque I: the axis of the applied torque passes through gripspace (e.g. using a screwdriver).

- Torque II: the axis of the applied torque passes outside of gripspace (e.g. pulling a lever).

The user study developed later in this paper is based on the four primary categories of this force-centric taxonomy. It will allow us to validate the use of the Virtual Mitten for these high-level categories of tasks that cover most interaction cases.

\subsection{Hand-based manipulation techniques}

Several egocentric 3D interaction techniques are based on hand gestures. For example, virtual pointing [1] lets users choose objects by aiming an input device or a finger at them. However, these techniques do not involve the true dynamics of grasping. The virtual hand metaphor [7] allows a more natural interaction by giving a virtual presence to the users' real hands in the simulation. One common control scheme in this case is to map the motion of the real hand to the motion of the virtual hand. Kry et al. [18] proposed an alternative control scheme in which the user has control over the folding/unfolding and global displacement of a virtual hand via rate-control by applying small displacements of each finger.

In the most basic form of the virtual hand metaphor, objects are glued to the hand upon contact but more elaborate implementations allow us to realistically grasp objects. For example, the hand can be composed of many collision points for physically-based grasping [13]. Another approach is to let heuristic rules govern the grasping and the release of an object by identifying valid grasping configurations from contact data $[29,20]$.

When the real hand is not physically constrained, collisions with the virtual environment cannot be perceived haptically. Thus, visual feedbacks have been devised in order to convey this information by constraining the visual representation of the hand. For that purpose, Jacobs adapted the principle of the god-object to the structure of the hand [16] and Borst proposed to use a physically simulated massspring system to link real and virtual hands [4].

Visual feedback is also used to graphically express the interaction forces occurring during manipulation tasks. For example, segments of the virtual hand can be colored to express a contact [2] or a valid grasping status [20]. In a broader context, Sreng et al. [27] proposed to use explicit glyphs and illumination effects to denote interaction forces and proximity.

\subsection{Active haptic feedback}

To transmit the forces occurring in a virtual environment to users, desktop-based haptic devices such as the Virtuose 6D (Haption SA, France) are available. While these devices allow us to move objects around a virtual environment, using them requires specific interaction metaphors that lack an operational symmetry with reality in that users do not perform real grasping motions. This limitation is inappropriate for training applications where gestures and tasks must be reproduced in silico in the same manner as in reality.

Other haptic devices are inherently conceived for finger-based manipulation. The MasterFinger-2 [21], for example, is made of two articulated arms, each one ending with a thimble to insert fingertips. By actuating the index and the thumb, it allows the reproduction of sensations related to pinching operations but suffers from a small workspace that prevents wide movements of the arm.

Hand-mounted haptic devices allow direct manipulation of objects through the fingers while giving users more freedom of movement than grounded devices. The Rutgers Master II [6] is a lightweight haptic device nested in the user's palm. Pneumatic actuators bound to the thumb and three fingers provide high forces but the system must still be linked through tubes to an air compressor. The CyberGrasp (CyberGlove Systems, USA) is a commercial haptic exoskeleton that actuates each fingertip by pulling a tendon passing through a sizable armature on the back of the hand. It can render grasping forces but its high cost is a barrier to a wider adoption.

\subsection{Alternative haptic feedback}

Alternative methods have been proposed to simulate haptic properties without active force-feedback. For example, passive haptic feedback leverages input devices that benefit from enough internal elasticity to let users get relevant haptic cues when deforming them themselves. Pihuit et al. [24] proposed an elastic device taking the form of a foam ball equipped with pressure sensors under each finger to perform sculpting tasks. Kry et al. [18] proposed the HandNavigator, another passive device that allows users to control a virtual hand by slightly displacing the fingers inside of deformable thimbles. The main limitation of both of these desktop devices is that users suffer from a small workspace. Hoang et al. [14] attached a block of conductive digital foam on the palm of a glove in order to measure pressure and provide passive haptic feedback as well as mobility. However, this system is not focused on object manipulation but rather on environment modeling with the palm pressed flat against shapable objects. Hummel et al. [15] proposed to grab objects via pinching motions. The lifting force is mapped from the pressure applied between two fingers, which provides a passive feedback from the user's own body and simulates heaviness.

Another alternative means of delivering haptic sensations is pseudo-haptic feedback, a category of methods that simulate haptic properties by relying on visual feedback coupled with the motion and actions of the user. Lécuyer et al. [19] coined the term with an experiment in which a virtual cube manipulated with a passive device slowed down when crossing a specific area. As a result, users tended to apply more force on the passive device to displace the 
cube and thus perceived more friction. Dominjon et al. [10] evaluated the potential of pseudo-haptic feedback for the perception of mass. The visual motion of virtual balls being manipulated via a passive haptic device was amplified or reduced vertically to express heaviness. Other physical properties such as torque [23] and softness [17] are expressible through pseudo-haptic feedback.

Passive haptic feedback and pseudo-haptic feedback are rich techniques that allow us to generate and modulate haptic cues without complex haptic devices. The Virtual Mitten paradigm relies on these methods to enhance grip-based interaction, as discussed in the next section.

\section{The Virtual Mitten}

In this section, we describe the different aspects of the Virtual Mitten as a visuo-haptic interaction paradigm for object manipulation. Firstly, we introduce its general concept before going into details about the elastic input device used, the associated control scheme, the visual feedbacks and the pseudo-haptic approach that simulates different levels of effort. Then, use-cases are presented in order to illustrate the capabilities of this novel interaction paradigm.

\subsection{Concept}

The proposed interaction paradigm is based at its core on a handheld elastic input device that maps the motion of the user's hand to a virtual mitten capable of interacting with virtual objects. The grasping of an object and the following interaction depends on the grip force applied on the device. Due to its internal elasticity, our device provides a passive force feedback and enables the perception of efforts in the context of manipulation tasks occurring within the virtual environment.

The elastic device used is simple in nature as well as low-cost. Nevertheless, it effectively provides relative haptic sensations enhancing grip-based interaction with virtual objects. Moreover, its small dimensions and its low weight preserve the freedom of movement of users within the virtual environment. The visual metaphor - a mitten with a generic folding animation - provides a natural mapping between real and virtual environments. Real hands and virtual mittens are not colocalized and extensions of the virtual hand metaphor (such as the go-go technique [7]) could thus be additionally applied to the Virtual Mitten.

Our interaction paradigm has four main components:

- an elastic input device that maps the position and grip force of the user's hand to a virtual mitten,

- a control scheme to naturally select and manipulate virtual objects with the virtual mitten,

- visual feedbacks graphically expressing the effort occurring during interaction,

- a pseudo-haptic feedback to modulate the perceived haptic sensations.

\subsection{Elastic input device}

For the prototyping of our interaction paradigm, a consumer-grade hand exerciser [25] that is both inexpensive and commonly available was used as an input device. Its low weight (62 grams), its small size $(8 \times 7 \times 1.5$ centimeters $)$ and its shape that perfectly fits the hand due to its original purpose are valuable features. Its inherent elasticity is another major feature since it allows passive haptic feedback. The system offers a stiffness of approximately 4400 N. $\mathrm{m}^{-1}$ over a range of 2 centimeters.

We engineered the device so that it could be coupled with an optical tracking system in order to retrieve its position and orientation in $3 \mathrm{D}$ space (6DOF) as well as its compression (1DOF). In our current implementation, the individual position of each finger is not tracked, which results in a device that is easier to track than a fully articulated hand while still being able to measure grasping motions.
Interaction in the virtual environment is modulated with respect to the grip force applied on the elastic device. This quantity is formalized as a normalized compression ratio $r$ such that $r=0$ when the device is relaxed and $r=1$ when the device is fully compressed. $r$ is computed from the area $A$ defined by four reflective markers (see Figure 2) and varies linearly with respect to the minimum area $A_{\min }$ (when the device is fully compressed) and the maximum area $A_{\max }$ (when relaxed) such that $r=1-\frac{A-A_{\min }}{A_{\max }-A_{\min }}$. For better mapping between real and virtual environments, the rest pose of the virtual mitten corresponds to the pose of the user's hand when the elastic device is relaxed.

The compression exerted on the elastic device by the user is not directly mapped to the compression within the simulation. Instead, a filtering is established with $r_{\text {real }}$ the compression ratio optically measured, $k$ a stiffness coefficient (empirically set to 0.15 in our prototype in order to provide both smoothing and responsiveness) and $r_{\text {virtual }}$ (or simply $r$ ) the output virtual compression ratio effectively used in the simulation (see Equation 1). This filtering flattens tracking inaccuracies and smooths the interaction with virtual objects.

$$
r_{t}=r_{\text {virtual }, t}=r_{\text {virtual }, t-1}+k \times\left(r_{\text {real }, t}-r_{\text {virtual }, t-1}\right)
$$

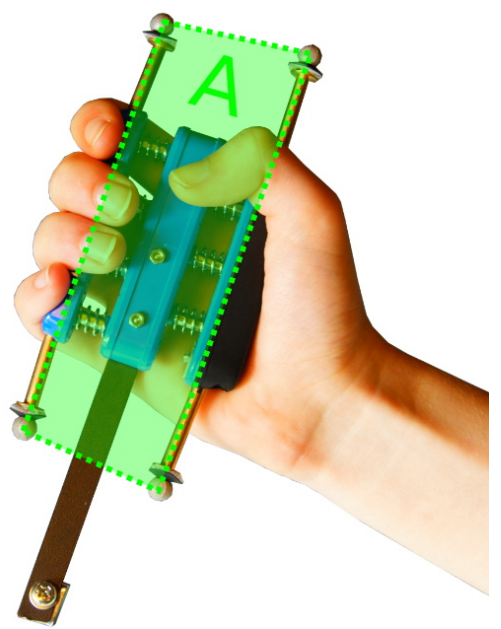

Figure 2: Our elastic input device is a consumer-grade hand exerciser equipped with markers for optical tracking. Here, the elastic device is fully relaxed so $A=A_{\max }$ (green overlay) and $r=0$.

\subsection{Control scheme}

The Virtual Mitten is associated to a control scheme that allows users to control mittens representing their own hands (both unimanual and bimanual scenarios are possible). This control scheme allows us to operate mittens in a unified manner that is truthful to the real dynamics of grasping: first, mittens are moved in space, then fingers come into contact with targeted objects and finally an adjustment of the grip-force ensures a secure grasp.

\subsubsection{Virtual coupling: Displacing the mitten in 3D space}

The mitten naturally follows the user's hand in 3D space: by moving his hand, he directly controls the position and orientation of the mitten. Since the virtual environment is physically simulated, it is thus possible to interact coarsely with virtual objects by touching or pushing them.

When considering the input device used, the arms of the user cannot be constrained by the virtual environment. In order to solve 


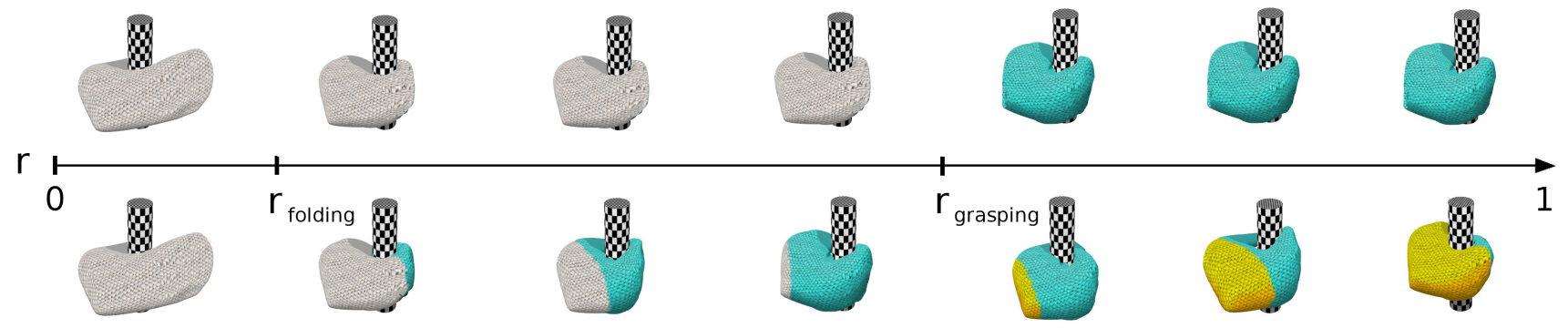

Figure 3: Different visual feedbacks proposed for displaying the evolution of the grip force when grasping a virtual cylinder. Above the axis: "Boolean" feedback. Below the axis: "Progressive" feedback. The $r_{\text {folding }}$ threshold is fixed while the $r_{\text {grasping }}$ threshold varies in order to accommodate our pseudo-haptic effect.

this issue, a virtual coupling is established (6DOF virtual spring [4]) between the virtual mitten and the user's real hand. This way, the virtual mitten follows the user's motion as closely as possible but will also slide along virtual surfaces. This virtual coupling also enhances the stability of the motion of the mitten by flattening the normal tremors of the arm.

\subsubsection{Object selection: Closing and opening the mitten}

When using the virtual hand metaphor in its most basic form, grasped objects are simply glued to the hand upon contact. With the Virtual Mitten, a naturalistic approach that reproduces the true dynamics of grasping is proposed. Indeed, to select an object for further interaction, the mitten has to be placed in a valid grasping configuration around the targeted object, that is, there must be at least one contact between thumb and object and at least one contact between merged fingers and object.

To validate this condition and bring the digits of the mitten closer to an object, the user has control over the clenching of the mitten. A finger folding animation is triggered when a slight compression of the device is detected ( $\left.r \geq r_{\text {folding }}\right)$. Conversely, when the device is relaxed $\left(r<r_{\text {folding }}\right)$, the hands initiates an unfolding animation. Once triggered, the speed of this animation is constant and does not vary with the compression.

This motion of the user's digits only uses a small part of the compression range of the elastic device since most of it is reserved for the manipulation part of the interaction. As such, a small $r_{\text {folding }}$ threshold must be chosen, low enough so that it does not take up too much spring length and large enough so that it does not unintentionally trigger the animation due to tracking inaccuracies or false positive detection of the user's intent (it is set to 0.05 in our prototype). Furthermore, it is crucial that the folding animation adapts to the shape of the considered objects to avoid interpenetration and maintain realism. To that effect, an incremental joint-unfolding method was used [5].

\subsubsection{Object manipulation: Using grip force for interacting through the mitten}

Once an object is selected, a condition related to the effort exerted on the elastic device must be fulfilled: users have to apply a sufficient amount of force on the elastic device to grasp the targeted object securely and not let it slip. More formally, the compression ratio $r$ must be greater than another fixed threshold $r_{\text {grasping }}$ so that the object is attached to the mitten. Once a virtual object has been grasped with the mitten, the exerted compression must be maintained above $r_{\text {grasping }}$ in order not to drop it.

Objects can thus be manipulated and moved around the virtual environment. Additionally, tools capable of executing actions benefit from an additional degree of freedom in that their inherent operation can be modulated with respect to the gripping force applied.
For example, the closing of a pair of scissors can be mapped to the remaining compression range such that the user has fine control over a paper cutting task.

\subsection{Visual feedbacks}

We propose two different visual feedbacks to graphically expose the amount of force exerted on the elastic device when grasping an object: a Boolean feedback and a Progressive feedback.

Boolean feedback. While the compression ratio is less than the $r_{\text {grasping }}$ threshold, the appearance of the mitten remains unchanged. However, when this grasping threshold is reached, the mitten turns to a different color (blue in our prototype) in order to indicate that the exerted force is sufficient to grasp the object (see Figure 3 - top).

Progressive feedback. While the compression ratio is less than $r_{\text {grasping }}$, the mitten is continuously filled from its tip to its base with a primary color (blue) representing the effort required to reach this threshold and grasp the object. When $r \geq r_{\text {grasping }}$, the mitten is continuously filled in the other direction with a secondary color (yellow) representing the excess of compression with respect to the grasping threshold (see Figure 3 - bottom). In other words, the mitten acts as a gauge displaying first the compression requirement for interaction and then the excess of compression.

\subsection{Pseudo-haptic feedback}

The Virtual Mitten, as described until this section, allows us to manipulate virtual objects and get a passive haptic feedback through the elastic device. However, the compression threshold $r_{\text {grasping }}$ that needs to be reached to grasp an object is constant, therefore the perceived effort is basically the same for each virtual object. We introduce here a novel pseudo-haptic approach in order to allow the perception of different levels of effort.

The general principle is that the higher the magnitude of the simulated haptic property is, the higher the $r_{\text {grasping }}$ threshold that has to be reached is. This pseudo-haptic feedback thus replaces the unique $r_{\text {grasping }}$ with object-specific $r_{\text {grasping }}$ (object) thresholds that associate a haptic property with a required grip-force. The appearance of our visual feedbacks (Boolean and Progressive) are correlated with these object-specific thresholds so that they demonstrate different dynamics depending on the targeted object. For example, when grasping an object with a higher threshold, the mitten would change color more slowly and the users would tend to apply more force to speed up the grasp. Due to the elasticity of our input device, this would induce stronger haptic cues.

The value of the $r_{\text {grasping }}$ threshold bound to each manipulable object is calculated via a context-based mapping function that takes as input the magnitude of the physical property being simulated. For example, when simulating heaviness, the masses of the interactive objects are considered and mapped to the $[0,1]$ range of the 


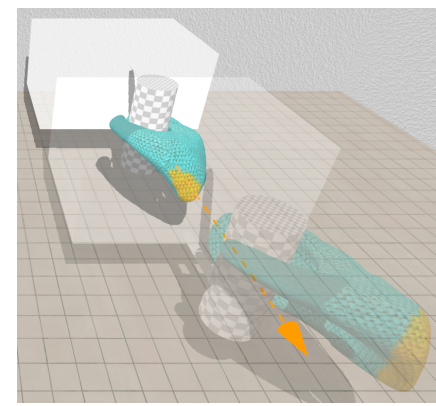

(a) Force I: Pulling a drawer.

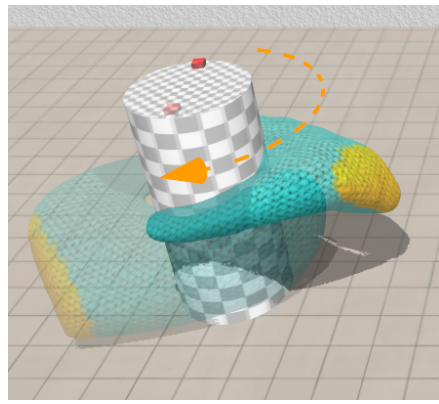

(c) Torque I: Screwing an object.

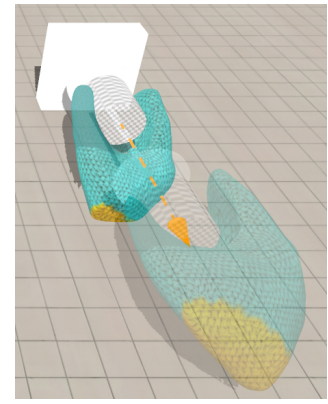

(b) Force II: Pulling a pin.

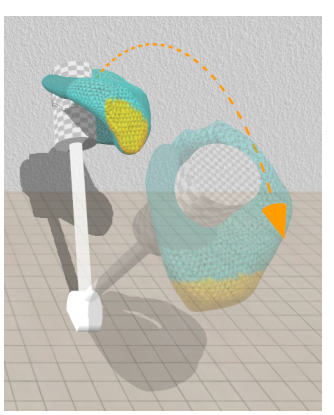

(d) Torque II: Pulling a lever.
Figure 4: Primitive haptic tasks corresponding to the taxonomy of Bloomfield et al. [3].

elastic device. Similarly, other haptic properties such as stiffness, friction or the effort to operate a tool can be simulated. To deliver an optimal pseudo-haptic feedback, the mapping function must consider the order of magnitude of all the interactive objects of the scene. Indeed, using a linear mapping in a scene populated with objects bearing a high difference of magnitude (feathers and cars) would incur a loss of resolution for the elastic device when comparing light objects between them whereas a non-linear mapping could enhance haptic perception.

\subsection{Illustrative use cases}

Taxonomy of haptic tasks. The first illustrative use cases address the four primitive categories of Bloomfield's taxonomy of haptic tasks [3] (see Figure 4).

For the Force I scenario, the simulated haptic property is the pulling of a drawer. The drawer can be opened by grasping its handle and performing a linear displacement of the hand. For the Force II scenario, the simulated haptic property is the stickiness of a pin stuck on a wall. At first glance, the Force I and Force II scenarios seem similar but when pulling the pin, grip-forces are perpendicular to the direction of motion (towards the head of the pin).

For the Torque I scenario, the simulated haptic property is the screwing of an object. The object can be screwed by grasping it and performing a rotation of the wrist. For the Torque II scenario, the simulated haptic property is the pulling of a lever. These two tasks both involve a rotation but the axis differs: for the screwing task, the axis is in grip-space while it is outside of grip-space for the lever task.

Fruit-o-Matic. Another illustrative scenario, that we called the Fruit-o-Matic, involves a series of primitive manipulation tasks (see Figure 5). The goal is to prepare fruit juice with a blender. Users are provided with two elastic devices so that both their hands can interact with the virtual environment. Various virtual objects can be grabbed and moved around with the mitten: fruits, the lid of the blender or a glass. Fruits can be squeezed above the blender to
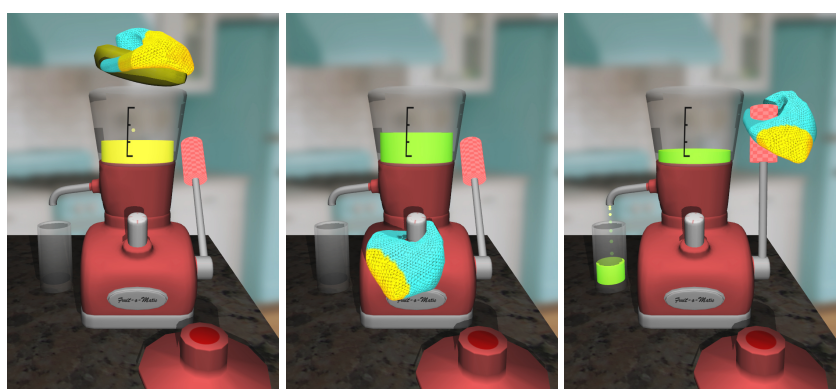

Figure 5: The "Fruit-o-Matic", an entertaining use-case. The preparation of a fruit juice involves sub-tasks such as grabbing objects, squeezing fruits, rotating a dial to operate the blender and pulling a lever to pour the juice.

extract their juice. Each fruit has a different internal stiffness and requires a different amount of effort to be pressed. A dial has to be turned to mix the juices. Finally, a lever must be pulled to pour the mixed juice into the glass.

\section{Evaluation}

The evaluation of the Virtual Mitten was decomposed in two different experiments: (1) a subjective evaluation to compare the two proposed visual feedbacks (Boolean versus Progressive) and to assess the appeal of the Virtual Mitten (2) a preliminary psychophysical evaluation in order to assess the users' perception and the resolution of our elastic device when simulating a screwing effort. The user study considered the tasks defined in Bloomfield's taxonomy [3] as depicted in Figure 4.

\subsection{Apparatus and participants}

Apparatus The environment was displayed on a 55 inches screen placed at 2 meters from participants. In order to allow users to rest their elbows while interacting and minimize muscular fatigue, users were seated in front of a table with a keyboard to answer the tests (see Figure 6).

The optical tracking system used was a Vicon Bonita system (Vicon, USA) with ten infrared cameras. The tracking data was further processed with the Vicon Nexus reconstruction software and streamed into our application which used OpenSceneGraph as a rendering engine and Bullet Physics for the physics simulation. It is most important to mention that this tracking setup does not describe the minimal requirement to use the Virtual Mitten since it would contradict the promise of a low-cost interaction paradigm. Thanks to the basic tracking model bound to the elastic device used, setups with simpler tracking solutions and pressure sensors to measure the compression of the device are also appropriate.

In order to avoid occlusions between the real user's hand and the visual content, the mitten was not colocalized with the hand of the user and an offset was introduced (the mitten was approximately 50 centimeters in front of the real hand). In addition, as the placement of the cameras was done to maximize the tracking accuracy of the user's hands and not the tracking of the head, we used monoscopic rendering from a static point of view.

Participants Twelve participants (males and right-handed) took part in the experiments, ranging from 21 to 28 years old $(M=$ $25.2 ; S D=2.6)$. Regarding their experience with virtual reality, three were experienced users, six had a moderate experience and three had no prior experience. No participants had any prior knowledge about the Virtual Mitten. Users started each experiment by a training session and could take breaks at any time. 


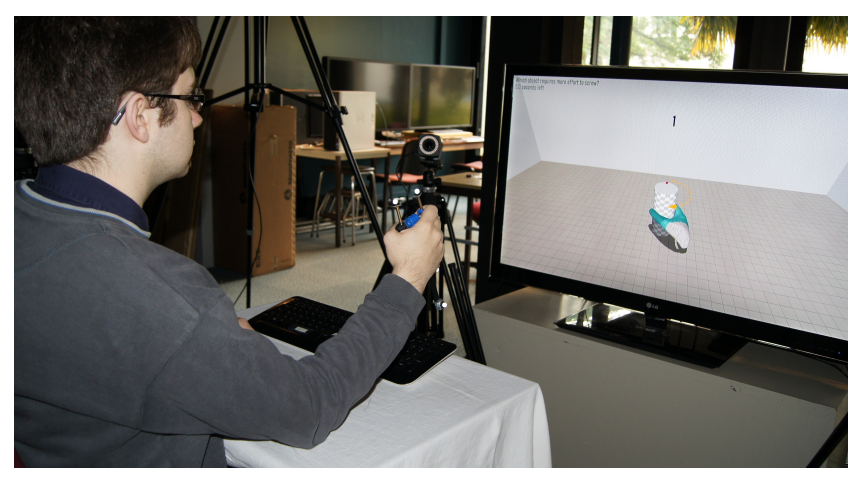

Figure 6: Experimental setup. Users are seated in front of a screen displaying the simulation. They can rest their arm on a table. A keyboard with marked keys allows them to enter their answers.

\subsection{Subjective Evaluation of the Virtual Mitten}

The first experiment was based on the four primitive haptic tasks defined by Bloomfield's taxonomy [3] so that differences between the two proposed visual feedbacks could be observed for various types of interaction.

Procedure Participants had to carry out classical Two Alternative Forced Choices (2AFC). For each trial, users had to perform two repetitions of a same task but each repetition was associated to a different $r_{\text {grasping }}$ threshold. Once the user had finished both interactions, he had to answer the question "Which task required more effort to perform?" using a keyboard and their answers were considered correct if they chose the interaction with the highest grasping threshold.

Design and Hypotheses The independent variables of the experiment were: the Task (Drawer, Pin, Screw, Lever), the Visual Feedback (Boolean, Progressive) and the Grasping Threshold (Soft, Hard). The soft grasping threshold $r_{\text {grasping }}$ (Soft) was 0.5 and the hard grasping threshold $r_{\text {grasping }}$ (Hard) was 0.625 . The thresholds were determined through informal evaluations, ensuring that users will be able to perceive the difference but with a certain level of error. While the four tasks were always presented in the same order, the ordering of the visual feedbacks and the ordering of the grasping thresholds were counterbalanced. Each Task/Visual Feedback combination was repeated 3 times, resulting in 24 comparisons per participant.

The dependent variables were the answers entered by the participants and the mean compression exerted by participants during each grasp. At the end of the evaluation, participants also indicated which visual feedback they preferred and answered the questions listed in Table 1 and 2 with a 7-point Likert scale.

The results from this evaluation will permit to validate the following hypotheses:

H1 Participants will provide more correct answers when the Progressive visual feedback is used.

H2 The mean compression will be lower for the Progressive feedback than for the Boolean feedback.

\subsection{Psychophysical assessment for the screwing task}

The second experiment of our user study focused on assessing the perceptual resolution of our elastic device for simulating a specific pseudo-haptic property with the Virtual Mitten. We have focused on one primitive haptic task from Bloomfield taxonomy: the screwing effort (Torque I). We have also restricted our study to the Progressive feedback which was found to be the preferred visual feedback in the first experiment.

Procedure A Just Noticeable Difference (JND) psychophysical study [12] is proposed to measure the minimum difference between two $r_{\text {grasping }}$ thresholds that can be discriminated by users and thus assess the resolution of the elastic device. Participants had to follow a $2 \mathrm{AFC}$ procedure: each trial was a comparison between a reference grasping threshold (fixed) and a comparison grasping threshold (from a set of precomputed thresholds). Participants had to answer the question "Which was the object requiring more effort to screw?".

Design The independent variable was the grasping threshold, with a reference value of $r_{\text {grasping }}($ Reference $)=0.45$ and six comparison thresholds $r_{\text {grasping }}($ Comparison $)=$ $0.288,0.342,0.396,0.504,0.558,0.612$. The comparison thresholds were computed as $r_{\text {grasping }}($ Reference $) \times(1+\Delta)$ with $\Delta \in\{-0.36,-0.24,-0.12,0.12,0.24,0.36\}$. The values of the reference and comparison thresholds serve as a baseline for this evaluation and were chosen empirically so that the covered range contains JND values already studied in the literature. For each trial, participants had to determine which condition (comparison versus reference) required more effort to perform. Each pair was repeated 5 times, resulting in 30 comparisons per participant.

\subsection{Results}

\subsubsection{Subjective evaluation results}

The mean compression applied by participants was analyzed using a repeated measures three-way ANOVA with the factors Task, Visual Feedback and Grasping Threshold. The data followed a normal distribution (Anderson-Darling test with a $p<0.05$ ). Regarding the post-hoc comparisons, we used Bonferroni pairwise tests adjusted for $\alpha=95 \%$. Only significant post-hoc comparisons are mentioned $(p<0.05)$. The three-way ANOVA showed a main effect for Visual Feedback $\left(F(1,11)=41.1, p<0.001, \eta_{P}^{2}=0.415\right)$ and Grasping Threshold $\left(F(1,11)=148.8, p<0.001, \eta_{P}^{2}=0.857\right)$. Bonferroni post-hoc tests confirm the main effects, showing differences among several levels. The mean compression is significantly lower for the Progressive feedback $(M=0.648 ; S D=0.091)$ compared to the Boolean feedback $(M=0.691 ; S D=0.077)$. Regarding the grasping threshold, post hoc-tests also showed significant differences among the two levels $r_{\text {grasping }}(\mathrm{Soft})(M=0.628 ; S D=0.09)$ and $r_{\text {grasping }}$ (Hard) $(M=0.710 ; S D=0.06)$. Figure 7 summarizes the mean compression for each visual feedback and each task.

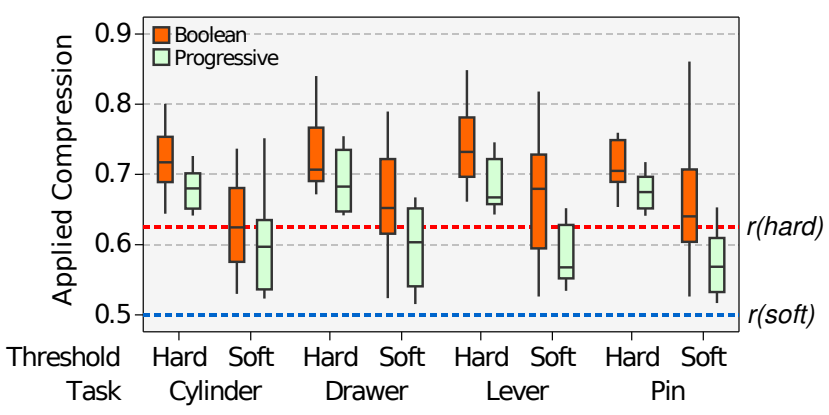

Figure 7: Boxplot for the mean applied compression grouped by task and grasping threshold.

The percentage of correct answers for each task considering the two Visual Feedback evaluated are displayed in Figure 8. Pairwise t-tests showed a significant difference for amount of correct answers between the Progressive feedback $(M=0.85 ; S D=0.19)$ and the Boolean feedback $(M=0.73 ; S D=0.29),(t(2)=-2.8 ; p<0.01)$. In contrast, pairwise t-tests did not show any significant differences among interaction tasks. Regarding potential learning effects, the analysis of the evolution of the mean applied compression and the answers of participants did not show any correlation. 


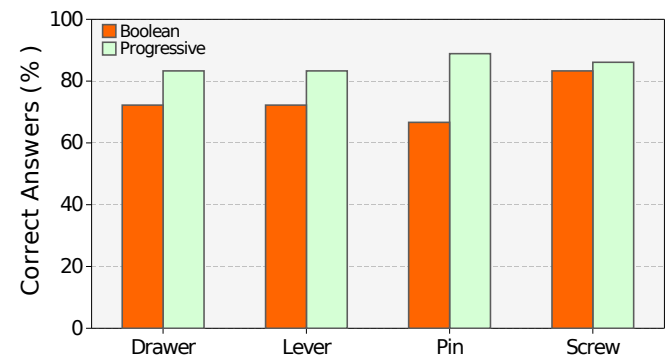

Figure 8: Mean percentage of correct answers for each visual feedback, grouped by task.

Concerning the questionnaire, we performed pairwise Wilcoxon's tests for the results of each question considering the four Tasks and the two Visual Feedbacks. Regarding Q1, two pairwise tests were significant: (1) "Pulling the drawer" vs "Pulling the Pin" $(p<0.01)$ and "Pulling the drawer" vs "Screwing the cylinder" $(p<0.01)$. Users found "Pulling the drawer" an easier task than "Pulling the Pin" and "Screwing the cylinder" (see Table 1). The analysis for Q2, Q3 and Q4 (see Table 2) did not show any significant difference.

\begin{tabular}{|l|c|c|}
\hline Q1: “Was this task easy to accomplish?” \\
\hline \hline Task & M & SD \\
\hline Pulling the drawer & 5.7 & 0.9 \\
\hline Pulling the pin & 4.8 & 1.05 \\
\hline Screwing the cylinder & 4.5 & 1.18 \\
\hline Pulling the lever & 5 & 1.6 \\
\hline
\end{tabular}

Table 1: Questionnaire results for the first question (7-point Likert scale).

\begin{tabular}{|l|c|c|}
\cline { 2 - 3 } \multicolumn{1}{c|}{ Q2: “Did you well perceive a difference between the two objects?” } & $\mathrm{M}$ & $\mathrm{SD}$ \\
\hline Q3: “Did you consider the haptic feedback realistic?" & 1.305 \\
\hline Q4: “Did you answer with confidence?" & 4.948 & 1.348 \\
\hline
\end{tabular}

Table 2: Questionnaire results for questions Q2, Q3 and Q4 (7-point Likert scale).

Concerning the user's preferences, 5 users preferred the Boolean feedback while 7 users preferred the Progressive feedback. The comments for each group of users are consistent. Users preferring the Boolean feedback state that it allows them to better focus on the task and not on the visual feedback (with the Boolean feedback: "I could focus on the task", "It made me act faster"). On the contrary, users who preferred the Progressive feedback state that it allows them to be more precise and adjust the level of force required to grasp an object ("the drop of the object is more predictable").

\subsubsection{Psychophysical assessment results}

The goal of the perceptual evaluation was to compute the Just Noticeable Differences (JND) between two grasping thresholds when performing a screwing task.

First, we computed the percentage of answers in which the repetition using the reference compression threshold was considered as the one requiring additional effort. As expected, as the value of $r_{\text {comparison }}$ decreases, the reference is chosen more often and vice versa. Then, using Weber's law, we computed the Weber Fraction (k) as $k=\Delta I / I$, where $\Delta I$ refers to the Just Noticeable Difference threshold and $I$ the grasping threshold for the reference. The JND threshold can be determined as the value of the stimuli in which the recognition ratio is $84 \%$ [12]. In order to compute the compression ratio for the recognition rate of $84 \%$, we fit the psychometric curve $f(x)=1 /\left(1+e^{(x+\alpha) / \beta}\right)$ to the data (Weibull function with $\alpha=0.0042$ and $\beta=0.0692$ ) (see Figure 9). Furthermore, we observe that the Point of Subjective Equality (the user provides a random answer) is consistent since the fit shows that it is approximately 0 . The Weber fraction of the compression ratio for the screwing task was $k=\Delta I / I=0.267$ which means that users are capable of discerning approximately four different levels of effort with the elastic device in this specific context.

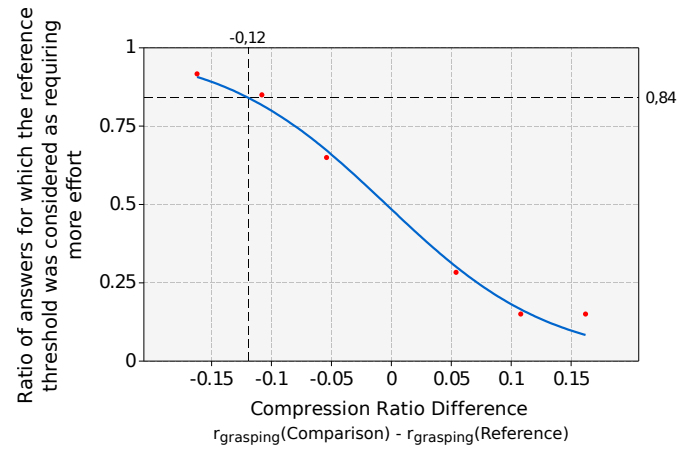

Figure 9: Psychometric curve found in second experiment. It plots the average percentage of answers in which participants considered the task using the reference threshold as the one requiring more effort to perform.

\section{Discussion}

The user study provided insight about the potential of our novel interaction paradigm both in terms of appeal and perception. Concerning the subjective evaluation, two visual feedbacks (Progressive and Boolean) were proposed and tested in the context of various manipulation tasks. The results are similarly good for the different tasks, which suggests that our paradigm can apply successfully to all contexts mentioned in Bloomfield's taxonomy [3]. For the comparison tasks, users gave a greater number of correct answers when using the Progressive visual feedback thus supporting hypothesis $\mathrm{H} 1$. The measure of the compression exerted by participants shows that their grasping is more precise with the Progressive feedback: the mean compression applied is closer to the grasping threshold associated with virtual objects, which validates hypothesis H2. This result is consistent with those of Fabiani et al. [11] who evaluated that a combination of visual and haptic feedback greatly helps in achieving precise grasps. However, in our case, these feedbacks are coupled via the pseudo-haptic approach and the simpler visual information relates to the whole mitten rather than individual fingers. The results on discrimination and precision are consistent thus the higher amount of correct answers could stem from the enhanced precision while grasping virtual objects. Indeed, a more precise compression implies a more precise haptic feedback due to the elastic nature of the input device.

However, even though performances were globally better with Progressive feedback, several participants preferred the Boolean feedback (42\%). The Boolean feedback was found to be less prone to distract the users and let them focus on the task. For future usage of the Virtual Mitten it seems thus preferable to give users the choice of their visual feedback. It also seems that visual feedback might be disturbing for the users. It might thus be interesting to discard the additional visual cues progressively after a learning period for more expert users.

Concerning the second evaluation, a psychophysical protocol was used to assess the perceptions of users in the specific context 
of a screwing task as a pilot experiment. The results of our series of discrimination trials enabled us to compute a psychometric function and to compute the corresponding Just Noticeable Difference, i.e., the discrimination threshold for the screwing effort. The final JND obtained is close to $26 \%$, which seems consistent with the JND values given in the haptic literature and corresponding to either force $(12 \%)$, torque $(16 \%)$ or stiffness $(22 \%)$ [9]. The lower resolution found here (i.e., higher JND) could be due to the fact that the perceived effort corresponds in our case to a more complex context involving a sequence of actions (selecting an object and then rotating it). This pilot experiment could be followed by other evaluations applying this psychophysical protocol to the three other categories from Bloomfield's taxonomy (namely, Force I, Force II and Torque II). Other evaluations could also follow in order to compare the Virtual Mitten with existing interaction techniques: users could be asked to perform various manipulation tasks sequentially with the Virtual Mitten and with other types of interfaces so that differences in performance (speed and precision) and in perception could be assessed.

\section{ConClusion}

The Virtual Mitten is a novel interaction paradigm for the manipulation of virtual objects. Our goal was to provide a new way to interact with virtual objects in a haptic manner that is faithful to the dynamics of grasping and do not require active haptic feedback or complex input devices. Our approach is based on the passive haptic feedback provided by a handheld elastic input device (an engineered hand-exerciser). The grip force exerted on the device enables to grasp objects and to achieve various manipulation tasks by means of a virtual mitten. A pseudo-haptic effect was also introduced to generate the haptic perception of different levels of effort.

A user study was conducted to assess the acceptance of our novel interaction paradigm by naive participants and the perception of the pseudo-haptic feedback. The results suggest first that the Virtual Mitten allows us to reliably manipulate virtual objects in various primitive manipulation tasks (pulling a drawer, pulling a pin, screwing a cylinder, pulling a lever). A psychophysical test showed that different levels of effort could be successfully perceived in a basic screwing task. These results confirm that our pseudo-haptic effect is well perceived by participants. An entertaining application (the Fruit-o-Matic) involving bimanual interaction and a sequence of manipulation tasks was also provided to illustrate the versatility of the Virtual Mitten.

Taken together, our results suggest that our novel interaction paradigm could be applied to various manipulation cases and used in multiple virtual reality applications in which a simple haptic information is important such as for virtual prototyping, sport training, rehabilitation procedures or video games.

Future work could first concern the design of other elastic input devices for other interaction possibilities. More accurate tracking systems could be used to access more haptic inputs. Multi-finger interaction could be studied by making full use of multi-finger versions of hand exercisers. Then, we could think of using other visual metaphors such as virtual pliers that seem well adapted to the shape and usage of our elastic input device. Finally, other evaluations could be considered to assess the scalability of our interaction paradigm in various tasks, such as for industrial assembly or maintenance simulations.

\section{ACKNOWLEDGEMENTS}

The authors thank Anthony Talvas and Jonathan Mercier-Ganady for their help. This work was supported by ANR (MANDARIN project, ANR-12-CORD-0011).

\section{REFERENCES}

[1] F. Argelaguet and C. Andujar. A Survey of 3D Object Selection Techniques for Virtual Environments. Computers \& Graphics, 37(3):121$136,2013$.
[2] M. Bergamasco, P. Degl'Innocenti, and D. Bucciarelli. A realistic approach for grasping and moving virtual objects. In Proc. of IEEE/RSJ IROS, pages $717-724$ vol.1, 1994.

[3] A. Bloomfield, Y. Deng, J. Wampler, P. Rondot, D. Harth, M. McManus, and N. I. Badler. A Taxonomy and Comparison of Haptic Actions for Disassembly Tasks. In Proc. of IEEE VR, pages 225-231, 2003.

[4] C. W. Borst and A. P. Indugula. Realistic Virtual Grasping. In Proc of IEEE VR, pages 91-98, 2005.

[5] R. Boulic, S. Rezzonico, and D. Thalmann. Multi-Finger Manipulation of Virtual Objects. In Proc. of ACM VRST, pages 67-74, 1996.

[6] M. Bouzit, G. V. Popescu, G. C. Burdea, and R. F. Boian. The Rutgers Master II-ND Force Feedback Glove. In Proc. of HAPTICS, pages 145-152, 2002.

[7] D. A. Bowman, E. Kruijff, J. J. LaViola, and I. Poupyrev. $3 D$ User Interfaces: Theory and Practice. 2004.

[8] I. M. Bullock and A. M. Dollar. Classifying Human Manipulation Behavior. In Proc. of IEEE ICORR, pages 1-6, 2011.

[9] G. C. Burdea. Force and Touch Feedback for Virtual Reality. 1996

[10] L. Dominjon, A. Lecuyer, J.-M. Burkhardt, P. Richard, and S. Richir. Influence of Control/Display Ratio on the Perception of Mass of Manipulated Objects in Virtual Environments. In Proc. of IEEE VR, pages 19-25, 2005.

[11] L. Fabiani, G. Burdea, N. Langrana, and D. Gomez. Human Interface Using the Rutgers Master II Force Feedback Interface. In Proc. of IEEE VRAIS, pages 54-59, 1996.

[12] G. A. Gescheider. Psychophysics : Method, Theory, and Application. Lawrence Erlbaum Associates, New Jersey, US, 1985.

[13] K. Hirota and M. Hirose. Dexterous object manipulation based on collision response. In Proc. of IEEE VR, pages 232-, 2003.

[14] T. N. Hoang, R. T. Smith, and B. H. Thomas. 3D Interactions with a Passive Deformable Haptic Glove. In Proc. of IEEE ISMAR, pages $1-6,2013$.

[15] J. Hummel, J. Dodiya, R. Wolff, A. Gerndt, and T. Kuhlen. An Evaluation of Two Simple Methods for Representing Heaviness in Immersive Virtual Environments. In Proc. of IEEE 3DUI, 2013.

[16] J. Jacobs, M. Stengel, and B. Fröhlich. A Generalized God-Object Method for Plausible Finger-Based Interactions in Virtual Environments. In Proc. of IEEE 3DUI, pages 43-51, 2012.

[17] T. Kimura and T. Nojima. Pseudo-haptic Feedback on Softness Induced by Grasping Motion. In Proc. of EuroHaptics, pages 202-205, 2012.

[18] P. G. Kry, A. Pihuit, A. Bernhardt, and M.-P. Cani. HandNavigator: Hands-on Interaction for Desktop Virtual Reality. In Proc. of ACM VRST, pages 53-60, 2008

[19] A. Lécuyer, S. Coquillart, A. Kheddar, P. Richard, and P. Coiffet. Pseudo-Haptic Feedback: Can Isometric Input Devices Simulate Force Feedback? In Proc. of IEEE VR, pages 83-90, 2000.

[20] M. Moehring and B. Froehlich. Effective manipulation of virtual objects within arm's reach. In Proc. of IEEE VR, pages 131-138, 2011.

[21] M. Monroy, M. Oyarzábal, M. Ferre, A. Campos, and J. Barrio. MasterFinger: Multi-finger Haptic Interface for Collaborative Environments. In Proc. of EuroHaptics, pages 411-419, 2008.

[22] J. R. Napier. The Prehensile Movements of the Human Hand. The Journal of Bone and Joint Surgery, 38-B:902-913, 1956.

[23] A. Paljic, J.-M. Burkhardt, and S. Coquillart. Evaluation of pseudohaptic feedback for simulating torque: a comparison between isometric and elastic input devices. In Proc. of HAPTICS, pages 216-223, 2004.

[24] A. Pihuit, P. G. Kry, and M.-P. Cani. Hands-on virtual clay. In Proc. of IEEE SMI, pages 267-268, 2008.

[25] Prohands. Gripmaster Light. www. prohands . net.

[26] M. Schlattman and R. Klein. Simultaneous 4 gestures 6 DOF realtime two-hand tracking without any markers. In Proc. of ACM VRST, pages 39-42, 2007.

[27] J. Sreng, A. Lécuyer, C. Mégard, and C. Andriot. Using Visual Cues of Contact to Improve Interactive Manipulation of Virtual Objects in Industrial Assembly/Maintenance Simulations. IEEE TVCG, 12(5):1013-1020, 2006.

[28] C. L. Taylor and R. J. Schwarz. The Anatomy and Mechanics of the Human Hand. Artificial limbs, 2:22-35, may 1955.

[29] T. Ullmann and J. Sauer. Intuitive Virtual Grasping for Non Haptic Environments. In Pacific Conf. on Computer Graphics and Applications, pages 373-380, 2000. 